\title{
Raf kinase inhibitory protein: a signal transduction modulator and metastasis suppressor
}

\author{
Alexey E Granovsky, Marsha Rich Rosner \\ Ben May Department for Cancer Research, University of Chicago, 929 East 57 $7^{\text {th }}$ Street, Chicago, IL 60637, USA
}

Cells have a multitude of controls to maintain their integrity and prevent random switching from one biological state to another. Raf Kinase Inhibitory Protein (RKIP), a member of the phosphatidylethanolamine binding protein (PEBP) family, is representative of a new class of modulators of signaling cascades that function to maintain the "yin yang" or balance of biological systems. RKIP inhibits MAP kinase (Raf-MEK-ERK), G protein-coupled receptor (GPCR) kinase and $N F \kappa B$ signaling cascades. Because RKIP targets different kinases dependent upon its state of phosphorylation, RKIP also acts to integrate crosstalk initiated by multiple environmental stimuli. Loss or depletion of RKIP results in disruption of the normal cellular stasis and can lead to chromosomal abnormalities and disease states such as cancer. Since RKIP and the PEBP family have been reviewed previously, the goal of this analysis is to provide an update and highlight some of the unique features of RKIP that make it a critical player in the regulation of cellular signaling processes.

Keywords: Raf Kinase, metastasis, signal transduction, inhibitor

Cell Research (2008) 18:452-457. doi: 10.1038/cr.2008.43; published online 1 April 2008

A key feature of establishing robustness is generating positive and negative feedback loops to bring systems back to their initial equilibrium states following perturbation. Raf Kinase Inhibitory Protein (RKIP or PEBP1) performs this function by responding to feedback signals that modulate its role as a direct negative regulator of key signaling kinases [1]. Identified as a Raf kinase inhibitor following 2-hybrid screening [2], RKIP had previously been characterized as a phospholipid binding protein. Mammalian RKIP/PEBP is distinct from other known proteins and its function is still being elucidated. In addition to binding phospholipids, RKIP has been reported to bind nucleotides and opioids [3]. However, our recent NMR studies have shown that most of these ligands do not bind to RKIP in solution at neutral $\mathrm{pH}$ (data not shown). The PEBP protein family comprises over 400 members, and crystallization of PEBPs from a variety of species, including bacteria, plant, bovine, rat and human, revealed that their basic structure as well as sequence are evolutionarily conserved ${ }^{[1]}$. A protein of appr. 25kDa size, the PEBPs have two notable features: a ligand binding pocket, and a compact globular structure that provides ample surface area for interaction with other proteins (Figure 1). These two properties are critical for

Correspondence: Marsha Rich Rosner

E-mail:m-rosner@uchicago.edu enabling RKIP to play a unique role as a regulator of cell stasis.

The structure of RKIP appears to inform its function. Mutations in plant and yeast PEBPs have highlighted the physiological importance of the ligand-binding pocket of RKIP. In plants, naturally occurring mutations in the PEBP homologs revealed a role in controlling the switch between shoot growth and flowering, a process involving vegetative growth versus reproductive development [4]. Mutations in the TERMINAL FLOWER1 (TF1) gene of Arabidopsis [5], the CENTRORADIALIS (CEN) gene in Antirrhinum [6] and the self-pruning gene in tomato plants (SP) [7]result in shorter shoots with terminal flowers. Conversely, overexpression of TFL1, CEN or SP prolongs the vegetative growth phase. Another member of the Arabidopsis RKIP/ PEBP family, FLOWERING LOCUS T (FLT), functions in an antagonistic fashion to block vegetative growth [8]. Of particular interest, a mutation in the conserved DPDxP motif of the pocket (P76L) induces a switch from shoot growth to flowering in tomato plants $[7,9]$. Comparable pocket mutations in the yeast PEBP, Tfs $1 p$, block its inhibition of Ras GAP (Ira2) by preventing their association [10]. We have recently shown that comparable mutation of the RKIP pocket at P74L (Figure 1) also blocks its inhibition of Raf-1 as measured by activation of ERK1 and ERK2, the Raf-1-regulated MAPKs, and prevents RKIP inhibition of 
EGF-stimulated DNA synthesis (Clark, et al., submitted). These results indicate that the role of the ligand-binding pocket in regulating PEBP/RKIP function is likely evolutionarily conserved among eukaryotes.

The specific steps involved in RKIP regulation of Raf signaling are not entirely clear. RKIP overexpression can inhibit MEK interaction with Raf-1 and B-Raf [11] but the extent to which this happens under physiological conditions is not known. However, using an siRNA approach to investigate the role of the endogenous protein, we demonstrated that RKIP blocks activation of Raf- 1 in cells by preventing access to activating kinases and phosphorylation at S338 and Y341, events required for Raf-1 activation [12]. In contrast, B-Raf activation was not regulated by RKIP. The role of RKIP in regulating Raf-1 function could indirectly affect B-Raf signaling via the Raf-1-B-Raf dimer, which is dependent upon 14-3-3, an adapter protein known to associate with Raf-1 in mammalian cells [13]. Studies of oncogenic B-Raf mutants from melanoma patients have shown that kinase-dead B-Raf can signal to MAPK via association with Raf-1 [14]. However, in Raf- $1^{-/}$MEFs, RKIP depletion can increase B-Raf-mediated MEK activity suggesting that RKIP can regulate B-Raf independent of Raf-1 [15]. Of note, a yeast two hybrid study using the tomato RKIP/PEBP homolog SP as bait identified several associated plant proteins including a serine/threonine kinase of the NIMA subfamily (SPAK) as well as an isoform of 14-3-3 [9]. Interestingly, SPAK binds both 14-3-3 and $\mathrm{SP}$ at the same site in a phosphorylation-dependent manner, and overexpression of 14-3-3 compensates for the $s p$ mutant defect, raising the possibility that the RKIP/PEBP family, like 14-3-3, may function as adapter proteins that promote complex formation or dimerize and sequester proteins.

How does RKIP physically interact with Raf-1? RKIP expression prevents phosphorylation of Raf-1 at S338 [16] and Y341 [17], and phosphomimetic mutations at these sites (S338D/Y340D/Y341D) block RKIP interaction with Raf-1, suggesting that this region is the site of Raf-1 interaction with RKIP [12]. Subsequent studies based on in vitro binding of RKIP to small peptides from the S338-Y341 Raf1 phosphorylation region are consistent with this possibility [18]. RKIP is a small globular protein that presumably can interact with the larger Raf-1 kinase at more than one site. One region of RKIP that appears to interact with Raf-1 is the ligand binding pocket since mutation of the pocket or pocket occupancy prevents RKIP association with Raf-1 and inactivates RKIP inhibitory function (Clark, et al., submitted). However, as noted above, phosphorylation of RKIP at S153 (Figure 1) by growth activating kinases such as Protein Kinase C also prevents RKIP interaction with Raf-1 [19]. Steric hindrance by the phosphate groups rather than charge repulsion is likely to be responsible, since a S153E mutant binds to Raf- 1 as effectively as wild type RKIP [19]. The S153 site is close to the loop region that lines the pocket. Thus, a model involving Raf-1 binding to the RKIP pocket and the neighboring region containing S153 would explain the results observed to date. However, since effects of mutations can be indirect, detailed structural studies will be required to elucidate the specific sites of RKIP-Raf-1 interaction.

RKIP functions as a modulator rather than an off switch
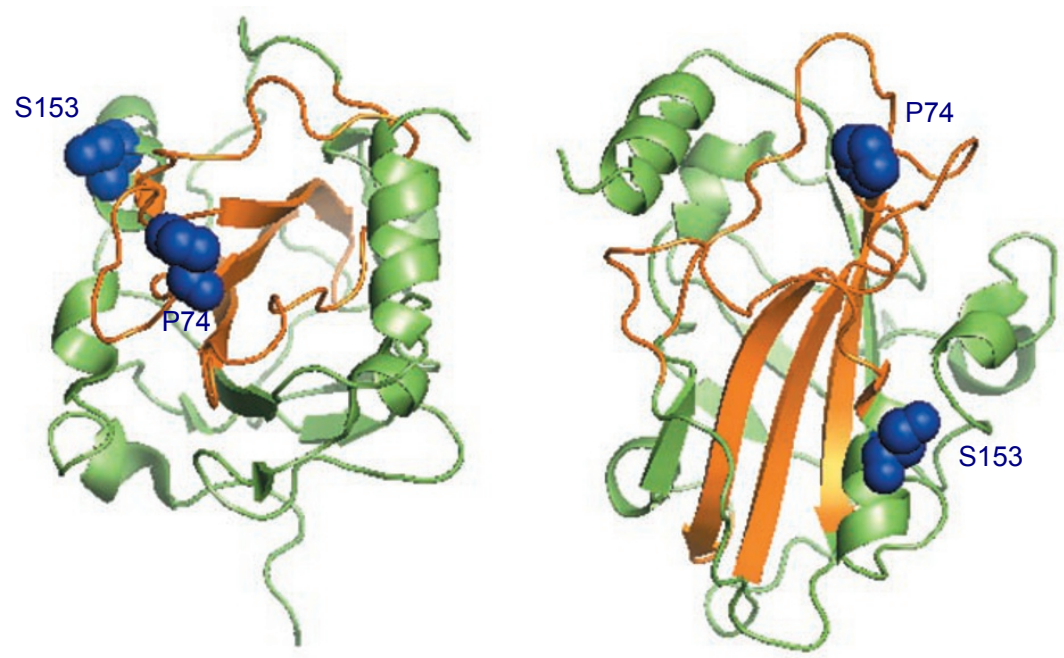

Figure 1 Two different cartoon representations of the x-ray crystal structure of rat RKIP (PDB Id\#2iqy). Residues comprising the ligand binding pocket are shown in orange. The site of a naturally occurring pocket mutation in the conserved DPDxP motif (Pro74) and the site of RKIP phosphorylation by growth activating kinases (Ser153) are shown as blue spheres. 
for MAP kinase signaling. RKIP depletion from cells does not induce signaling in the absence of a stimulus nor change the overall kinetics of the response. Instead, its role is to tamp down the resultant amplitude of the output in response to stimuli such as EGF. Depletion of RKIP induces an increase in the signal by approximately 2 -fold, enabling a shift in the EGF dose response of 5-10 fold for both MAP kinase activation and DNA synthesis [12]. Loss of RKIP could therefore influence the sensitivity of the system to environmental factors and theoretically impact on development, a process that is often dependent upon a gradient of activating or inhibitory factors. To preserve the integrity of the system, why not eliminate RKIP and just limit the potential amplitude of the MAPK signal? The answer presumably lies in the ability of RKIP to respond to and integrate multiple environmental stimuli. For example, Protein Kinase C (PKC) activated by EGF, TPA or other regulatory receptor systems phosphorylates RKIP at S153, enabling RKIP-Raf-1 dissociation and inactivating RKIP's function as a Raf-1 inhibitor [19]. However, phosphorylation of RKIP at S153 enhances its ability to bind and inhibit G protein-coupled receptor kinase 2 (GRK2), a kinase that phosphorylates and down-regulates many $\mathrm{G}$ proteincoupled receptors (GPCRs) including the 32 -adrenergic receptor [20]. Thus, RKIP is an environmental sensor and integrator that "switches" from down-regulation of the MAPK cascade to potentiation of GPCR signaling. RKIP may maintain the system normally in an equilibrium state but is able to respond to an outside signal by transiently up- or down- regulating these pathways.

Another example of RKIP's role in maintaining cellular robustness is its regulation of the mammalian cell spindle checkpoint. The localization of phosphoS153 RKIP at centrosomes and kinetochores led us to investigate whether RKIP plays a role during mitosis. Subsequently, we showed that loss of RKIP or up-regulation of Raf kinase causes cells to move faster through mitosis from nuclear envelope breakdown to anaphase [21]. Furthermore, RKIP depletion and Raf-1 up-regulation override the Taxol-induced mitotic arrest caused by loss of spindle tension. The detailed mechanism involves bypass of the spindle checkpoint via inhibition of Aurora B kinase. Aurora B kinase, which localizes on inner centromeres in prometaphase in association with other "chromosomal passenger" proteins (INCENP, Survivin, Borealin/Dasra B [22]), is required to fix the errors in spindle-chromosome connections in order to ensure proper chromosome segregation during anaphase. A similar mitotic phenotype was observed upon direct inhibition of Aurora B kinase in Taxol-treated cells [23, 24]. The decrease in Aurora B kinase activity in response to RKIP depletion resulted from up-regulated MAP kinase. The specific target of MAPK is not clear since Aurora B kinase is not a direct substrate. More recently, we have evidence that Aurora B kinase participates in a negative feedback loop by phosphorylating RKIP at S153, causing dissociation of RKIP from Raf-1 and MAPK up-regulation (data not shown). The regulation by RKIP of the spindle checkpoint and genomic stability via the Raf-1/MAPK cascade [21] represents a mechanism that could explain in part its role in metastatic tumor progression.

The role of RKIP in preventing chromosomal abnormalities could contribute to its function as a suppressor of metastasis. RKIP loss or depletion is associated with metastatic disease in an increasing number of solid tumors. Initially, RKIP loss was identified as a prognostic marker for prostate cancer [25]. RKIP is depleted in distant metastases for both mismatch repair proficient and deficient colorectal cancers [26] and is a prognostic marker for disease-free survival [27]. Analysis of breast and melanoma cancer tissue showed a decrease in RKIP expression in lymph node metastases [28, 29]. RKIP is expressed in pancreatic islet cells, and absent or depleted in insulinomas [30]. Finally, decreased RKIP expression was noted in anaplastic thyroid tumors [31] and hepatocellular carcinoma tissue [32]. In a xenograft mouse model for prostate cancer, exogenous RKIP expression suppressed invasion and metastasis, and this reduction correlated with Raf-1 inhibition [33]. Specifically, overexpression of RKIP in human metastatic prostate cancer cells (C4-2B) that were implanted into the dorsal lobe of the prostate in mice decreased development of lung metastases and vascular invasion. In addition, RKIP overexpression in C4-2B cells led to reduced cell invasion in vitro. Interestingly, a Merkel carcinoma line was identified that overexpressed RKIP and lost ERK signaling; however, further studies revealed that RKIP depletion did not rescue the ERK signaling defect consistent with RKIP's role as a modulator rather than suppressor of the MAPK pathway [34]. Finally, a recent study showed that Snail, a mediator of the epithelial-mesenchymal transition (EMT), can inhibit RKIP transcription and negatively correlates with RKIP levels in tumors, consistent with a role for RKIP in metastasis [35]. Since RKIP expression is decreased during progression in a variety of cancers, it is likely that RKIP affects a fundamental step in the process rather than targeting tumor-specific mechanisms of invasion or colonization. Ultimately, animal studies involving either mouse cancer models in an $R K I P^{-/-}$background or human xenografts will be required to validate the role of RKIP in metastasis and the mechanism by which it regulates cancer progression.

A variety of functions relating to growth and differentiation have been ascribed to members of the PEBP family [1] and some of these phenotypes derive from mutations in different species including yeast and plants. Notably, as mentioned above, a theme of Ras/G protein signaling 
regulation is shared between the yeast and mammalian homologues. RKIP/PEBP has been shown to inhibit serine proteases, namely thrombin, neuropsin and chymotrypsin, although it shares no homology to any of the known families of serine protease inhibitors [36]. This function is reminiscent of that of the yeast PEBP, tfs $1 \mathrm{p}$, an inhibitor of Carboxypeptidase $\mathrm{Y}$ and regulator of PKA signaling [37]. RKIP also promotes human keratinocyte differentiation [38] and is induced during dendritic cell and macrophage differentiation [39]. Amino acids 2-12 of RKIP are identical to the hippocampal cholinergic neurostimulatory peptide (HCNP) that stimulates acetylcholine synthesis in rat septal nuclei and an enzyme capable of cleaving RKIP/PEBP to form HCNP has been detected in rat hippocampi [40]. RKIP has been found associated with plaques from Alzheimer's patients and may contribute to the disease process [41]. RKIP has also been reported to regulate the NFKB signaling cascade. Exogenously expressed RKIP can associate with NIK and TAK 1 and inhibit TNF $\alpha$ and IL- $1 \beta$ activation of NFkB [42]; RKIP can also potentiate apoptosis induced by chemotherapeutic agents in breast and prostate cancer cells [43, 44]. More recently, overexpressed RKIP was shown to inhibit Yin Yang 1 (YY1) expression in prostate and melanoma tumor cells, leading to induction of the DR5 death receptor and sensitizing the cells to TRAILmediated apoptosis [45]. Surprisingly, RKIP regulation of $\mathrm{NF \kappa B}$ and sensitivity to death ligands was not observed in nontumor cells, raising questions regarding the generality of this effect and whether the potentiation of death results indirectly from RKIP overexpression. To confuse matters more, hPEBP4, a related family member, was reported to block TRAIL and TNF $\alpha$-induced apoptosis even though it also inhibits Raf/MAPK signaling [46]. Therefore the normal physiological role of the PEBP family in regulating immune surveillance by the TNF $\alpha$ family is presently unclear. The availability of RKIP knockout mouse models and derived cells should help address the role of RKIP in cytokine signaling. However, the results to date raise the possibility that RKIP depletion in metastatic cells promotes the survival and propagation of solid tumor cells by enhancing resistance to normal immune surveillance.

Recently, the mouse PEBP1 homologue was targeted using a gene trap line of ES cells from the Sanger Institute, and the knockout was characterized [47]. The depletion of mPEBP1 was not lethal, and the phenotypes observed were relatively modest. A small deficit in odor-sensing was noted although the mechanism is unclear. Interestingly, a potential role for RKIP in olfaction has been suggested for its Drosophila homologs [48]. Several Drosophila PEBP isoforms have been identified from an antennal cDNA library, and four of these genes are expressed in hairs of the third antennal segment, which serves as an olfactory organ. Another gene is also expressed in taste organs in the head. Based on the expression patterns and sequence similarities to pheromone-binding proteins it has been suggested that PEBPs could be involved in odorant binding. Analysis of testicular germ cells indicated that RKIP is a sperm receptor for decapacitation factor, and RKIP deletion led to reduced reproduction rates and small litter size [49]. The modest phenotype might reflect the role of RKIP as a modulator. Alternatively, other PEBPs may compensate for loss of mouse PEBP1 due to redundancy in function. For example, mouse PEBP2, which is expressed exclusively in the testis [50] may prevent a more severe phenotype in testicular cells. Finally, mice have a number of processed PEBP pseudogenes that might also play a physiological role. The availability of mouse genetic models will enable these putative functions of RKIP and other PEBPs to be tested more rigorously.

Although more functions of RKIP will undoubtedly be elucidated, the evidence to date indicates that depletion or mutation of RKIP has major impact on the robustness of key biological signaling cascades involving growth factor, $\mathrm{G}$ protein and cytokine signaling. Whereas extreme perturbations are likely to lead to cell death, the more subtle changes elicited by loss of RKIP function cause defects such as checkpoint slippage that are not severe enough to destroy cells but are sufficient to move them to a disease state. Understanding the mechanisms that regulate RKIP interaction with its substrates will help us design therapeutic agents that can either potentiate or block RKIP function and promote tumor cell death either by inducing mitotic catastrophe or inhibiting key cellular cascades. Although this review has focused on RKIP (PEBP1), it is likely that other members of this ubiquitous family as well as other protein modulators perform similar roles in maintaining cellular integrity and homeostasis.

\section{Acknowledgments}

Work from the author's laboratory was supported by grants from the National Institutes of Health (NS33858 and CA112310 to M.R.R), the University of Chicago Cancer Research Center (to M.R.R.) and a gift from the Cornelius Crane Trust for Eczema Research (M.R.R.).

\section{REFERENCES}

1 Trakul N, Rosner MR. Modulation of the MAP kinase signaling cascade by Raf kinase inhibitory protein. Cell Res 2005; 15:1923.

2 Yeung K, Seitz T, Li S, et al. Suppression of Raf-1 kinase activity and MAP kinase signalling by RKIP. Nature 1999; 401:173177.

3 Schoentgen F, Jolles P. From structure to function: possible 
biological roles of a new widespread protein family binding hydrophobic ligands and displaying a nucleotide binding site. FEBS Lett 1995; 369:22-26.

4 Simister PC, Banfield MJ, Brady RL. The crystal structure of PEBP-2, a homologue of the PEBP/RKIP family. Acta Crystallogr D Biol Crystallogr 2002; 58 (Pt 6 Pt 2):1077-1080.

5 Shannon S, Meeks-Wagner DR. A Mutation in the Arabidopsis TFL1 Gene Affects Inflorescence Meristem Development. Plant Cell 1991; 3:877-892.

6 Bradley D, Ratcliffe O, Vincent C, Carpenter R, Coen E. Inflorescence commitment and architecture in Arabidopsis. Science 1997; 275:80-83.

7 Pnueli L, Carmel-Goren L, Hareven D, et al. The SELF-PRUNING gene of tomato regulates vegetative to reproductive switching of sympodial meristems and is the ortholog of CEN and TFL1. Development 1998; 125:1979-1989.

8 Ratcliffe OJ, Amaya I, Vincent CA, et al. A common mechanism controls the life cycle and architecture of plants. Development 1998; 125:1609-1615.

9 Pnueli L, Gutfinger T, Hareven D, et al. Tomato SP-interacting proteins define a conserved signaling system that regulates shoot architecture and flowering. Plant Cell 2001; 13:2687-2702.

10 Chautard H, Jacquet M, Schoentgen F, Bureaud N, Benedetti H. Tfs $1 p$, a member of the PEBP family, inhibits the Ira2p but not the Ira1p Ras GTPase-activating protein in Saccharomyces cerevisiae. Eukaryot Cell 2004; 3:459-470.

11 Yeung K, Janosch P, McFerran B, et al. Mechanism of suppression of the Raf/MEK/extracellular signal-regulated kinase pathway by the raf kinase inhibitor protein. Mol Cell Biol 2000; 20:3079-3085.

12 Trakul N, Menard RE, Schade GR, Qian Z, Rosner MR. Raf kinase inhibitory protein regulates Raf-1 but not B-Raf kinase activation. J Biol Chem 2005; 280:24931-24940.

13 Rushworth LK, Hindley AD, O’Neill E, Kolch W. Regulation and role of Raf-1/B-Raf heterodimerization. Mol Cell Biol 2006; 26:2262-2272.

14 Wan PT, Garnett MJ, Roe SM, et al. Mechanism of activation of the RAF-ERK signaling pathway by oncogenic mutations of B-RAF. Cell 2004; 116:855-867.

15 Park S, Yeung ML, Beach S, Shields JM, Yeung KC. RKIP downregulates B-Raf kinase activity in melanoma cancer cells. Oncogene 2005; 24:3535-3540 .

16 King AJ, Sun H, Diaz B, et al. The protein kinase Pak3 positively regulates Raf-1 activity through phosphorylation of serine 338 . Nature 1998; 396:180-183.

17 Fabian JR, Daar IO, Morrison DK. Critical tyrosine residues regulate the enzymatic and biological activity of Raf-1 kinase. Mol Cell Biol 1993; 13:7170-7179.

18 Park S, Rath O, Beach S, et al. Regulation of RKIP binding to the N-region of the Raf-1 kinase. FEBS Lett 2006; 580:64056412.

19 Corbit KC, Trakul N, Eves EM, et al. Activation of Raf-1 Signaling by Protein Kinase $\mathrm{C}$ through a Mechanism Involving Raf Kinase Inhibitory Protein. J Biol Chem 2003; 278:13061-13068.

20 Lorenz K, Lohse MJ, Quitterer U. Protein kinase C switches the Raf kinase inhibitor from Raf-1 to GRK-2. Nature 2003; 426:574-579.

21 Eves EM, Shapiro P, Naik K, et al. Raf kinase inhibitory protein regulates aurora $\mathrm{B}$ kinase and the spindle checkpoint. Mol Cell 2006; 23:561-574.
22 Vagnarelli P, Earnshaw WC. Chromosomal passengers: the fourdimensional regulation of mitotic events. Chromosoma 2004; 113:211-222.

23 Ditchfield C, Johnson VL, Tighe A, et al. Aurora B couples chromosome alignment with anaphase by targeting BubR1, Mad2, and Cenp-E to kinetochores. J Cell Biol 2003; 161:267-280.

24 Hauf S, Cole RW, LaTerra S, et al. The small molecule Hesperadin reveals a role for Aurora B in correcting kinetochoremicrotubule attachment and in maintaining the spindle assembly checkpoint. J Cell Biol 2003; 161:281-294.

25 Fu Z, Kitagawa Y, Shen R, et al. Metastasis suppressor gene Raf kinase inhibitor protein (RKIP) is a novel prognostic marker in prostate cancer. Prostate 2006; 66:248-256.

26 Minoo P, Zlobec I, Baker K, et al. Loss of raf-1 kinase inhibitor protein expression is associated with tumor progression and metastasis in colorectal cancer. Am J Clin Pathol 2007; 127:820827.

27 Al-Mulla F, Hagan S, Behbehani AI, et al. Raf kinase inhibitor protein expression in a survival analysis of colorectal cancer patients. J Clin Oncol 2006; 24:5672-5679.

28 Hagan S, Al-Mulla F, Mallon E, et al. Reduction of Raf-1 kinase inhibitor protein expression correlates with breast cancer metastasis. Clin Cancer Res 2005; 11:7392-7397.

29 Schuierer MM, Bataille F, Hagan S, Kolch W, Bosserhoff AK. Reduction in Rafkinase inhibitor protein expression is associated with increased Ras-extracellular signal-regulated kinase signaling in melanoma cell lines. Cancer Res 2004; 64:5186-5192.

30 Zhang L, Fu Z, Binkley C, et al. Raf kinase inhibitory protein inhibits beta-cell proliferation. Surgery 2004; 136:708-715.

31 Akaishi J, Onda M, Asaka S, et al. Growth-suppressive function of phosphatidylethanolamine-binding protein in anaplastic thyroid cancer. Anticancer Res 2006; 26 (6B):4437-4442.

32 Lee HC, Tian B, Sedivy JM, Wands JR, Kim M. Loss of Raf kinase inhibitor protein promotes cell proliferation and migration of human hepatoma cells. Gastroenterology 2006; 131:12081217.

33 Fu Z, Smith PC, Zhang L, et al. Effects of raf kinase inhibitor protein expression on suppression of prostate cancer metastasis. J Natl Cancer Inst 2003; 95:878-889.

34 Houben R, Ortmann S, Becker JC. RKIP does not contribute to MAP kinase pathway silencing in the Merkel Cell Carcinoma cell line UISO. J Carcinog 2007; 6:16.

35 Beach S, Tang H, Park S, et al. Snail is a repressor of RKIP transcription in metastatic prostate cancer cells. Oncogene 2007 Oct 22; doi: 10.1038/sj.onc. 1210860 .

36 Hengst U, Albrecht H, Hess D, Monard D. The phosphatidylethanolamine-binding protein is the prototype of a novel family of serine protease inhibitors. $J$ Biol Chem 2001; 276:535-540.

37 Caesar R, Blomberg A. The stress-induced Tfs $1 p$ requires NatB-mediated acetylation to inhibit carboxypeptidase $\mathrm{Y}$ and to regulate the protein kinase A pathway. J Biol Chem 2004; 279:38532-38543

38 Yamazaki T, Nakano H, Hayakari M, et al. Differentiation induction of human keratinocytes by phosphatidylethanolamine-binding protein. J Biol Chem 2004; 279:32191-32195.

39 Schuierer MM, Heilmeier U, Boettcher A, et al. Induction of Raf kinase inhibitor protein contributes to macrophage differentiation. Biochem Biophys Res Commun 2006; 342:1083-1087.

40 Ojika K, Kojima S, Ueki Y, et al. Purification and structural analysis of hippocampal cholinergic neurostimulating peptide. 
Brain Res 1992; 572:164-171.

41 Chen Q, Wang S, Thompson SN, Hall ED, Guttmann RP. Identification and characterization of PEBP as a calpain substrate. $J$ Neurochem 2006; 99:1133-1141.

42 Yeung KC, Rose DW, Dhillon AS, et al. Raf kinase inhibitor protein interacts with NF-kappaB-inducing kinase and TAK1 and inhibits NF-kappaB activation. Mol Cell Biol 2001; 21:72077217.

43 Chatterjee D, Bai Y, Wang Z, et al. RKIP sensitizes prostate and breast cancer cells to drug-induced apoptosis. J Biol Chem 2004; 279:17515-17523.

44 Jazirehi AR, Vega MI, Chatterjee D, Goodglick L, Bonavida B. Inhibition of the Raf-MEK1/2-ERK1/2 signaling pathway, Bcl$\mathrm{xL}$ down-regulation, and chemosensitization of non-Hodgkin's lymphoma B cells by Rituximab. Cancer Res 2004; 64:71177126.

45 Baritaki S, Katsman A, Chatterjee D, et al. Regulation of tumor cell sensitivity to TRAIL-induced apoptosis by the metastatic suppressor Raf kinase inhibitor protein via Yin Yang 1 inhibition and death receptor 5 up-regulation. J Immunol 2007; 179:5441-
5453.

46 Li H, Wang X, Li N, et al. hPEBP4 resists TRAIL-induced apoptosis of human prostate cancer cells by activating Akt and deactivating ERK1/2 pathways. J Biol Chem 2007; 282:49434950.

47 Theroux S, Pereira M, Casten KS, et al. Raf kinase inhibitory protein knockout mice: expression in the brain and olfaction deficit. Brain Res Bull 2007; 71:559-567.

48 Pikielny CW, Hasan G, Rouyer F, Rosbash M. Members of a family of Drosophila putative odorant-binding proteins are expressed in different subsets of olfactory hairs. Neuron 1994; 12:35-49.

49 Moffit JS, Boekelheide K, Sedivy JM, Klysik J. Mice Lacking Raf Kinase Inhibitor Protein-1 (RKIP-1) Have Altered Sperm Capacitation and Reduced Reproduction Rates With a Normal Response to Testicular Injury. J Androl 2007; 28:883-890.

50 Hickox DM, Gibbs G, Morrison JR, et al. Identification of a novel testis-specific member of the phosphatidylethanolamine binding protein family, pebp-2. Biol Reprod 2002; 67:917-927. 